\title{
Triazenos e atividade antibacteriana
}

\author{
Manfredo Hörner ${ }^{1}$, Vinícius Feltrin Giglio ${ }^{1}$, Aline Joana Rolina Wohlmuth Alves dos Santos ${ }^{1}$, \\ André Bilibio Westphalen', Bernardo Almeida Iglesias', Paulo Roberto Martins', Carlos Henrique \\ do Amaral' ${ }^{1}$, Tiago Mozaquatro Michelot ${ }^{2}$, Luiz Gustavo Brenner Reetz ${ }^{2}$, Cláudia de Mello \\ Bertoncheli $^{2}$, Gustavo Luiz Paraginski ${ }^{2}$, Rosmari Horner ${ }^{2 *}$
}

'Departamento de Química, Universidade Federal de Santa Maria, ²Departamento de Análises Clínicas e Toxicológicas, Universidade Federal de Santa Maria

"Correspondência:

R. Hörner

Departamento de Análises Clínicas e Toxicológicas

Universidade Federal de Santa Maria 97105-970 - Santa Maria - RS, Brasil. E-mail: rosmari@smail.ufsm.br
Quinze compostos triazenos foram estudados quanto à atividade antibacteriana pela metodologia de microdiluição em caldo. A Concentração Inibitória Mínima (CIM) e a Concentração Bactericida Mínima (CBM) foram pesquisadas frente a três bactérias padrão (E. coli ATCC 25922, Staphylococcus aureus ATCC 25923, Pseudomonas aeruginosa ATCC 27853) e frente a cepas hospitalares (Acinetobacter baumannii, Acinetobacter lwoffii, Ralstonia pickettii, Bordetella bronchiseptica, Micrococcus $s p$., Enterococcus sp., Staphylococcus epidermidis, Staphylococcus saprophyticus, Streptococcus agalactiae, Bacillus cereus, Corynebacterium $s p$., Rhodococcus $s p$., Salmonella $s p$., Serratia marcescens, Morganella morganii, Enterobacter cloacae, Shigella flexneri, Shigella sonnei, Shigella $s p$., Klebsiella pneumoniae, $E S B L$ Klebsiella oxytoca $14, E S B L$ Klebsiella pneumoniae 23, ESBL Klebsiella pneumoniae 24, ESBL Klebsiella pneumoniae 25, ESBL Escherichia coli 26, ESBL Klebsiella pneumoniae 27, ESBL Klebsiella pneumoniae 31, ESBL Escherichia coli 32, ESBL Klebsiella pneumoniae 37 e ESBL Escherichia coli 38). A maior atividade foi evidenciada para o composto 1-metil-3-(pcarboxifenil)triazeno 1-óxido (2) contra Streptococcus agalactiae $(C I M=16 \mu \mathrm{g} / \mathrm{mL}$ e $C B M=32 \mu \mathrm{g} / \mathrm{mL})$. Os compostos 1-fenil-3(4-nitrofenil)triazeno-1-óxido (9), 1-(4-nitrofenil)-3-(4carboxifenil)triazeno (10) e 1-(4-acetilaminofenil)-3-(4carboxifenil)triazeno (11) apresentaram CIMs de 32 a $64 \mu \mathrm{g} / \mathrm{mL}$ frente $a \mathrm{~S}$. edipermidis, $\mathrm{S}$. saprophyticus, Corynebacterium $s p$. $e$ E. cloacae. Os compostos 1-metil-3-feniltriazeno-1-óxido (1) , bis1,3-(4-acetiloxima)triazeno (3), bis-1,3 (4-acetilfenil)triazeno (4), 1-(2-fluorfenil)-3-(4-acetilfenil)triazeno (5), 1,3-(3-hidroxidifeniltriazenido)(piridil)(bis-oxo-vanádio) (12), 1-(3-nitrofenil)3-feniltriazeno (14), 1-(4-nitrofenil)-3-benziltriazeno (15) apresentaram CIM $=128 \mu \mathrm{g} / \mathrm{mL}$ frente a $\mathrm{S}$. aureus ATCC 25923, P. aeruginosa $A T C C 27853$, A. lwoffii, Micrococcus $s p$., S. epidermidis, S. saprophyticus, Corynebacterirum $s p$., E. cloacae, S. flenneri e S. sonnei. Os compostos 1-fenil-3-(4-acetilfenil)triazeno (6), 1,3-bis-(4-etoxicarbonilfenil) triazeno (7) e 3-(4carboxilatofenil)-1-metiltriazeno 1-óxido de potássio tetraidratado (13) apresentaram CIMs iguais ou maiores que $128 \mu \mathrm{g} / \mathrm{mL}$. Estes resultados demonstraram a potencial atividade biológica destes compostos contra bactérias Gram-positivas e Gram-negativas.
Unitermos

- Triazenos/ atividade antibacteriana

- Método de microdiluição em caldo 


\section{INTRODUÇÃO}

Triazenos (TZC) constituem uma classe de compostos químicos que possuem uma diversificada e comprovada atividade biológica. Apresentam atividade antibacteriana frente a bactérias Gram-positivas e Gram-negativas e atividade antifúngica frente a fungos leveduriformes e filamentosos (Shealy et al., 1966; Goswami, Purohit, 2001; Santos, 2005; Giglio, 2006; Paraginski, 2006). Entre os compostos que apresentam atividade contra bactérias e fungos, pode-se citar: o metil 5-(3,3-dimetil-1-triazeno)imidazol-4-carboxilato, que apresentou atividade antifúngica frente a Candida albicans ATCC 752, Hansenula wingei NRRL Y-2340, Saccharomyces carlsbergensis ATCC 9080, Rhodothorula $s p$. SRI 263, Venturia inequalis ATCC 11096, Penicillium roqueforti ATCC 6987, Phycomyces nitens ATCC 9984, Aspergillus flavus ATCC 9643, e atividade antibacteriana frente a $P$. aeruginosa ATCC 8709, Salmonella thyphimurium ATCC 7823, Shigella sonnei ATCC 9290, Bacillus subtilis ATCC 10744, S. aureus ATCC 6538 e Mycobacterium phalei ATCC 354 (Shealy et al., 1966); o composto 3-hidroxi-3- $p$-toluil-1-o-carboxifeniltriazeno, ativo frente a $E$. faecalis, $S$. aureus penicilina resistente $(2500$ unidades) e Trichophyton mentagrophytes; o composto 3hidroxi-3-o-toluil-1-o-carboxifeniltriazeno, frente a $E$. faecalis, $S$. aureus penicilina resistente (2500 unidades), Trichophyton mentagrophytes e Aspergillus fumigatus; o composto 3-hidroxi-3-m-toluil-1-o-carbóxifeniltriazeno frente a Trichophyton mentagrophytes e Sporotrix schenckii; os compostos 3-hidroxi-3-o-toluil-1-pcloroifeniltriazeno, 3-hidróxi-3-o-toluil-1-o-cloroifeniltriazeno, 3-hidróxi-3-m-toluil-1-feniltriazeno, 3-hidróxi-3$m$-toluil-1-p-feniltriazeno e 3-hidroxi-3-m-toluil-1-oclorofeniltriazeno mostraram atividade contra Trichophyton mentagrophytes; o composto 3-hidróxi-3-m-toluil-1-pclorofeniltriazeno foi ativo frente a Candida albicans, Cryptococcus neoformans e Trichophyton mentagrophytes; o composto 3-hidroxi-3-m-toluil-1- $p$-nitrofeniltriazeno, frente a Sporotrix schenkii e Trichophyton mentagrophytes; o composto 3-hidroxi-3-n-propil-1-p-clorofeniltriazeno, frente a Trichophyton mentagrophytes e Sporotrix schenckii e 3hidróxi-3-n-metil-1-(2,4,6-tribromofenil)triazeno, frente a Cryptococcus neoformans, Sporotrix schenckii, Trichophyton mentagrophytes e Aspergillus fumigatus (Goswami, Purohit, 2001). Ainda, são tripanossomicidas como o Berenil ${ }^{\circledR}$ (Pilch et al., 1995), antineoplásicos como a Dacarbazina $^{\circledR}$ (ASERCIT ${ }^{\circledR}$ ), utilizada no tratamento do melanoma maligno metastásico e na doença de Hodgkin (Peori, Vaughan, Hooper, 1998; Carvalho et al., 2000; Sanada, Takagi, Sekiguchi, 2004). Os TZC apresentam ainda, a atividade de nuclease, recentemente identificada, sendo ap- tos a clivar o DNA, tanto o genômico como o plasmidial (Hörner, 2003; Paraginski et al., 2006).

O nome triazeno tem sido utilizado para descrever uma classe de compostos de cadeia aberta que contém três átomos de nitrogênio interligados em seqüência $(\mathrm{N}=\mathrm{NN})$. São geralmente sintetizados por diazotação de uma amina primária aromática, seguido do acoplamento do sal de diazônio correspondente com uma amina secundária, formando o produto desejado com rendimento considerável (Zhao et al., 1997; Hörner et al., 2002; Hörner et al., 2003; Hörner et al., 2004; Hörner et al., 2006a,b).

O objetivo deste trabalho foi avaliar a atividade antibacteriana de 15 novos compostos triazenos inéditos obtidos por síntese, com determinação da concentração inibitória mínima (CIM) e concentração bactericida mínima (CBM), utilizando cepas padrão (American Type Culture Collection - ATCC) e cepas hospitalares, algumas delas apresentando multirresistência aos antimicrobianos comercialmente disponíveis.

\section{MATERIAL E MÉTODOS}

\section{Compostos utilizados}

Os compostos triazenos foram sintetizados conforme metodologia descrita na literatura (Zhao et al., 1997; Hörner et al., 2002; Hörner et al., 2003; Hörner et al., 2006a,b).

Os complexos foram obtidos a partir de uma solução contendo o composto triazeno e o sal em questão, em proporções estequiométricas (Zhao et al., 1997; Hörner et al., 2002; Hörner et al., 2003; Hörner et al., 2006a,b).

\section{METODOLOGIA}

Para a caracterização dos compostos foi utilizado um aparelho Mel-Temp II para determinação dos pontos de fusão, um espectrofotômetro Nicolet - Magna IR 760 para os espectros de absorção no infravermelho, um espectrofotômetro 1650 PC Shimadzu para os espectros UV/Visível e um difratômetro Bruker X8 Kappa Apex II para a elucidação das estruturas moleculares utilizando o método de difração de Raios-X em monocristal.

\section{Microrganismos}

As cepas bacterianas utilizadas foram armazenadas a $-20{ }^{\circ} \mathrm{C}$ no banco de microrganismos do Laboratório de Bacteriologia (LAB) do DACT, UFSM, RS, Brasil. Os microrganismos testados constituíram-se das cepas ATCC Gram-positivas, Staphylococcus aureus ATCC 25923, 
Gram-negativas, E. coli ATCC 25922 e Pseudomonas aeruginosa ATCC 27853, e cepas isoladas em culturas de diferentes espécimes clínicos como sangue, líquor, urina, lavado broncoalveolar, entre outros, obtidos no Hospital Universitário de Santa Maria (HUSM), constituídas pelas bactérias Gram-positivas Micrococcus sp., Enterococcus sp., S. epidermidis, S. saprophyticus, $S$. agalactiae, $S$. pyogenes, B. cereus, Corynebacterium sp. e Rhodococcus sp., e pelas Gram-negativas $A$. baumannii, $A$. lwoffii, $R$. pickettii, B. bronchiseptica, Salmonella sp., $S$. marcescens, M. morganii, E. cloacae, S. flexneri, S sonnei, Shigella sp., K. pneumoniae, ESBL $K$ oxytoca 14, ESBL $K$. pneumoniae 23, ESBL $K$. pneumoniae 24, ESBL $K$. pneumoniae 25, ESBL E. coli 26, ESBL $K$. pneumoniae 27, ESBL K. pneumoniae 31, ESBL E. coli 32, ESBL K. pneumoniae 37 e ESBL E. coli 38 . Entre as bactérias provenientes de isolamentos clínicos, algumas apresentavam multirresistência aos antimicrobianos testados, como já foi citado anteriormente, e são representadas pelas cepas de $E$. cloacae e os bacilos Gram-negativos produtores de betalactamases de espectro ampliado (ESBL). O perfil de sensibilidade aos antimicrobianos foi realizado pelo Laboratório de Análises Clínicas (LAC) do HUSM, setor de Bacteriologia, utilizando a automação (MicroScan - DADE BHERING) e concomitantemente no LAB-DACT, utilizando metodologia manual através da disco-difusão em ágar (Kirby-Bauer).

\section{Atividade antimicrobiana}

A atividade antibacteriana foi determinada para os compostos e seus precursores utilizando o método de microdiluição, baseado no documento M7-A6 do Clinical and Laboratory Standards Institute (CLSI - antigo National Comittee for Clinical Laboratory Standards - NCCLS). Este método é denominado "microdiluição", e envolve o uso de pequenos volumes de caldo Mueller Hinton colocados em placas de plástico estéreis retangulares com 96 poços, de fundo redondo.

Anteriormente à realização do teste, as cepas bacterianas foram ativadas, com dois subcultivos em ágar sangue (S. agalactiae, S. pyogenes e Corynebacterium sp.) e ágar Mueller Hinton (demais bactérias). O inóculo bacteriano foi preparado com turbidez idêntica a de uma solução padrão da Escala de McFarland, grau 0,5 (1 - 2x $10^{8} \mathrm{UFC} / \mathrm{mL}$ ). Esta suspensão foi diluída em solução salina e utilizada nos ensaios até 15 minutos após sua preparação, de maneira que cada poço contivesse aproximadamente $5 \times 10^{5} \mathrm{UFC} / \mathrm{mL}$. As placas foram incubadas por 24 horas a $35^{\circ} \mathrm{C} \pm 2{ }^{\circ} \mathrm{C}$. Após o período de incubação a CIM foi determinada visualmente, como a menor concentração dos compostos que inibiu completamente o crescimento dos microrganismos nos poços de microdiluição. A Concentração Bactericida Mínima (CBM) foi definida como a menor concentração capaz de inibir o crescimento destas subculturas. Cada ensaio foi realizado em triplicata.

Todos os compostos estudados e seus precursores foram solubilizados em etanol: água na proporção 1:2 ou 1:3. Inicialmente, foram preparadas soluções estoques dos triazenos na concentração $2560 \mu \mathrm{g} / \mathrm{mL}$. Essas soluções quando aplicadas nas placas foram diluídas 20 vezes na concentração mais alta testada $(128 \mu \mathrm{g} / \mathrm{mL})$. A partir daí, foram feitas diluições na razão de 2 para os demais poços. Assim, foram testadas 10 concentrações (128 a 0,25 $\mu \mathrm{g} / \mathrm{mL})$. Essas concentrações correspondem às concentrações preconizadas pelo CLSI para determinação da CIM para a maioria dos antibacterianos. O etanol (solvente) ficou na concentração máxima de 2,5\%. Foram realizados controles do solvente a fim de verificar a possível atividade do etanol sobre as bactérias testadas, controle positivo (somente meio de cultura e inóculo) e controle negativo (somente meio de cultura). Nesta concentração utilizada o etanol não apresentou atividade frente às bactérias analisadas. Ampicilina foi utilizada como antimicrobiano controle.

\section{RESULTADOS E DISCUSSÃO}

A pureza de cada triazeno foi controlada por meio de Espectrometria na região do Infravermelho (IR), comprimento de onda máximo $(\lambda)$ de absorção na região do Ultravioleta-Visível (UV/Vis), características físicas, bem como pela comparação dos espectros UV/Vis dos triazenos com os espectros deles contaminados com seus precursores. Esta comparação espectral pode ser visualizada na Figura 1 com o espectro do composto 1-metil-3- $(p-$ carboxifenil)triazeno 1-óxido (1) e com o espectro dele contaminado com quantidades mínimas de seus precursores ( ácido $p$-aminobenzóico e $N$-metil-hidroxilamina hidrocloreto. (Os dados dos outros compostos não são apresentados neste trabalho).

Na Tabela 1 estão apresentadas as estruturas dos compostos triazenos que foram utilizados para os testes antibacterianos e seus respectivos nomes químicos.

Os resultados da atividade antibacteriana dos compostos estão apresentados nas Tabelas II e III, respectivamente, com as CIMs obtidas com as cepas ATCC e as dos isolados clínicos.

Para testar a atividade antibacteriana dos 15 compostos triazenos elegeu-se 34 cepas de microrganismos, sendo três ATCC e 31 isolados clínicos. Foram incluídos 18 gêneros, com diferentes padrões de patogenicidade e de sensibilidade aos agentes antimicrobianos disponíveis. 


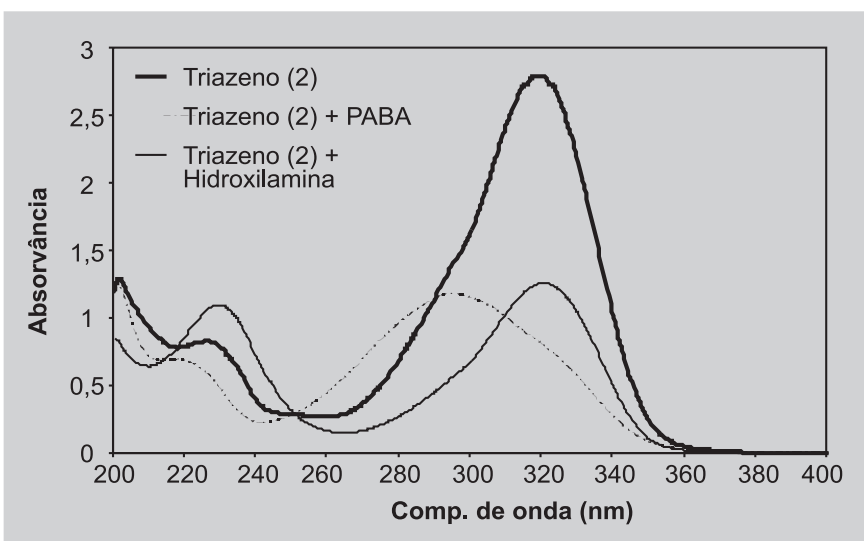

FIGURA 1 - Espectro no UV-Vis do triazeno (2), em etanol contaminado com quantidade mínima de ácido $p$-aminobenzóico (PABA) e de $N$-metil-hidroxilamina hidrocloreto na região de 200 a $500 \mathrm{~nm}$.

Micrococcus sp., Bacillus sp., Corynebacterium sp.(não produtor de toxina), Rhodococcus sp., Pseudomonas aeruginosa, Acinetobacter sp., Ralstonia pichettii e Bordetella bronchiseptica constituem patógenos oportunistas, que freqüentemente causam infecções em pacientes imunodeprimidos (Murray et al., 2003). Os membros da Família Enterobacteriaceae são importantes patógenos humanos, especialmente em ambientes hospitalares, onde causam os mais variados tipos de infecções, como infecções do trato urinário (Carson, Narber, 2004; Trautner, Daurowch, 2004), pneumonias (Zar, Cotton, 2002), meningites (Parodi et al., 2003) e septicemias (Takahashi et al., 2004), entre outras. A patogenicidade e crescente resistência dessa família a vários antimicrobianos é, atualmente, preocupante (Sader et al., 2001). Algumas enterobactérias, como Salmonella sp. e Shigella sp. estão sempre associadas a doenças em seres humanos, enquanto outras como $E$. coli e $K$. pneumoniae são membros da microbiota normal e podem causar infecções oportunistas (Brasil, 2004). Esta família de bactérias é de grande importância no ambiente hospitalar, não apenas por seus fatores de virulência, mas porque podem desenvolver resistência a várias classes de antimicrobianos. Finalmente, foram incluídas 10 cepas de bactérias ESBL, as quais representam um dos mais importantes problemas de resistência bacteriana nos hospitais brasileiros (Pereira et al., 2005). São bactérias capazes de produzir beta-lactamases que inativam os antibióticos betalactâmicos.

Dos quinze compostos triazenidos testados, 12 apresentaram algum tipo de atividade antibacteriana (Tabelas II e III), sendo que para nenhum dos seus precursores foi evidenciada tal ação (dados não apresentados nesse trabalho). Em relação ao espectro de ação, cinco compostos apresen- taram atividade antibacteriana de largo espectro $[(\mathbf{3}),(\mathbf{5}),(\mathbf{9})$, (10), e (11)], e os outros sete [(1), (2), (4), (8), (12), (14) e (15)] apresentaram atividade de espectro reduzido, tendo sido ativos ou frente a cepas Gram-positivas ou frente a Gram-negativas. A vantagem dos antibióticos de espectro reduzido é que eles podem ser utilizados no tratamento de infecções específicas sem interferir na população bacteriana normal protetora do hospedeiro (Murray et al., 2004). Em relação à concentração, dos compostos triazenos (TZC) estudados, os mais ativos foram o composto (2), com CIM =16 $\mu \mathrm{g} / \mathrm{mL}$ frente a $S$. agalactiae, e os TZC (9), (10), e (11), com $\mathrm{CIM}=32 \mu \mathrm{g} / \mathrm{mL}$ frente a $E$. cloacae e CIM $=64 \mu \mathrm{g} / \mathrm{mL}$ frente a Rhodococcus sp. Ainda, o composto (10) apresentou CIM $=64 \mu \mathrm{g} / \mathrm{mL}$ frente a $S$. saprophyticus e o composto (11), CIM de $64 \mu \mathrm{g} / \mathrm{mL}$ frente a S. epidermidis. Assim, o composto com melhor atividade antimicrobiana frente às bactérias testadas (CIM $=16 \mu \mathrm{g} / \mathrm{mL})$ foi o 1-metil-3-(4carboxifenil)triazeno-1-óxido (2), e o que apresentou atividade sobre o maior número de cepas (7 bactérias) foi o 1-(4nitrofenil)-3-(4-carboxifenil)triazeno (10). Nenhum dos compostos foi ativo frente às cepas ESBLs, que são em geral microrganismos multirresistentes. Atualmente, os carbapenêmicos vêm sendo utilizados para o tratamento de infecções por enterobactérias, principalmente o imipenem (Coudron, Hanson, Climo, 2003; Jones, Pfaller, 2003; Pagani et al., 2003).

A atividade do composto 1-metil-3-(4-carboxifenil)triazeno-1-óxido (2) contra S. agalactiae é importante, uma vez que é recomendada a quimioprofilaxia para gestantes que estiverem colonizadas, entre a trigésima quinta a trigésima sétima semana de gravidez, no trato genital/anal por esta bactéria, para prevenir a infecção do neonato, pois ela pode constituir-se no agente etiológico de septicemia, pneumomia e meningite em recém-nascidos, bem como a responsabilidade por doenças graves em adultos imunodeprimidos (Jackson et al., 1995; Schuchat, 1998).

A atividade encontrada frente a Enterobacter cloacae pelos compostos 1-fenil-3-(4-nitrofenil)triazeno1-óxido (9), 1-(4-nitrofenil)-3-(4-carboxifenil)triazeno (10), 1-(4-acetilaminofenil)-3-(4-carboxifenil)triazeno (11) é da mesma forma importante, uma vez que este microrganismo, juntamente com Citrobacter freundii, Morganella morganii e Serratia sp., constituem a causa mais comum de infecções hospitalares em neonatos e imunocomprometidos. Este grupo de bactérias acima citadas são freqüentemente resistentes a múltiplos antibióticos e juntamente com Pseudomonas aeruginosa fazem parte do grupo de produtores de beta-lactamases AmpC induzíveis (CESP) (Livermore, 1991; Sanders, Sanders, 1997; Murray et al., 2004).

Dos triazenos avaliados neste estudo, somente o 
TABELA I - Estruturas químicas dos compostos triazenos testados e seus respectivos nomes químicos

$$
R_{1}-N^{-}=N_{\substack{N \\ N}}-R_{2}
$$

Nome Químico

(1) 1-metil-3-feniltriazeno-1-óxido

(2) 1-metil-3-(4-carboxifenil)triazeno-1-óxido

(3) bis-1,3-(4-acetiloxima)triazeno

(4) bis-1,3-(4-acetilfenil)triazeno

(5) 1-(2-flúorfenil)-3-(4-acetilfenil)triazeno

(6) 1-fenil-3-(4-acetilfenil)triazeno

(7) 1,3-bis-(4-etoxicarbonilfenil)triazeno

(8) 1-(4-etoxifenil)-3-(4-carbóxifenil)triazeno

(9) 1-fenil-3-(4-nitrofenil)triazeno-1-óxido

(10) 1-(4-nitrofenil)-3-(4-carboxifenil)triazeno

(11) 1-(4-acetilaminofenil)-3-(4-carboxifenil)triazeno

(12) 1,3-(3-hidroxi-difeniltriazenido)

(piridil)(bis-oxo-vanádio)(v)

(13) Complexo 3-(4-carboxilatofenil)-1-metiltriazeno 1-óxido de potássio tetraidratado

(14) 1-(3-nitrofenil)-3-feniltriazeno

(15) 1-(4-nitrofenil)-3-benziltriazeno
$\mathrm{R}$

$\mathrm{OH}$

$\mathrm{OH}$

$\mathrm{H}$

$\mathrm{H}$

H

$\mathrm{H}$<smiles>Cc1ccccc1</smiles>

$\mathrm{H}$<smiles>CCOC(=O)c1ccc(C)cc1</smiles>

H<smiles>CCOc1ccc(C)cc1</smiles>

$\mathrm{H}$

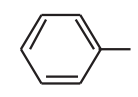

H<smiles>Cc1ccc([N+](=O)[O-])cc1</smiles>

H<smiles>Cc1ccc(C(N)=O)cc1</smiles>

Ver estrutura abaixo

$\mathrm{CH}_{3}$

$\mathrm{H}$

$\mathrm{H}$
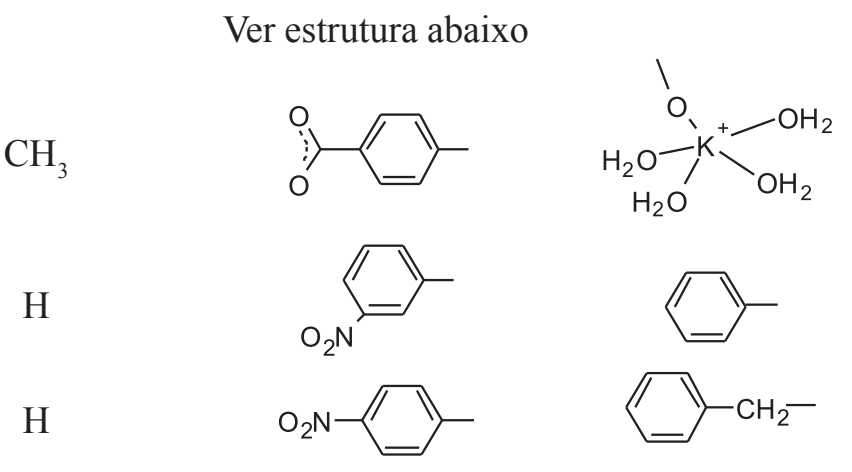

$\mathrm{CH}_{3}$<smiles>C</smiles><smiles>CC(=NO)c1ccc(C)cc1</smiles><smiles>CC(=O)c1ccc(C)cc1</smiles><smiles>CC(=O)c1ccc(C)cc1</smiles><smiles>CCC(=O)c1c#cc(C)cc1</smiles><smiles>CCOC(=O)c1ccc(C)cc1</smiles><smiles>Cc1ccc(C(=O)O)cc1</smiles><smiles>Cc1ccc([N+](=O)[O-])cc1</smiles><smiles>Cc1ccc(C(=O)O)cc1</smiles><smiles>Cc1ccc(C(=O)O)cc1</smiles><smiles>O=[PH](=O)(O)N1NN(c2ccccc2)ON(c2ccccc2)O1</smiles> 
TABELA II - Atividade antibacteriana in vitro dos compostos triazenos frente às cepas bacterianas ATCCs

\begin{tabular}{|c|c|c|c|c|c|c|c|c|c|c|c|c|c|c|c|}
\hline Cepas bacterianas & $(1)^{\mathrm{a}}$ & (2) & (3) & (4) & $\begin{array}{l}\text { Concer } \\
\text { (5) }\end{array}$ & $\begin{array}{r}\text { ação } \\
(6)\end{array}$ & $\begin{array}{l}\text { ibitór } \\
\text { (7) }\end{array}$ & $\begin{aligned} & \operatorname{míni1} \\
&(\mathbf{8})\end{aligned}$ & $\begin{array}{l}\text { (MIC } \\
(9)\end{array}$ & $\begin{array}{l}\mathrm{gg} / \mathrm{mL} \\
(\mathbf{1 0})\end{array}$ & (11) & (12) & (13) & (14) & (15) \\
\hline E. coli ATCC 25922 & $-{ }^{b}$ & - & - & - & - & - & - & - & - & 128 & - & - & - & - & - \\
\hline $\begin{array}{l}\text { Pseudomonas } \\
\text { aeruginosa ATCC } 27853\end{array}$ & - & - & - & - & - & - & - & - & - & - & - & - & - & 128 & - \\
\hline
\end{tabular}

a 1-metil-3-feniltriazeno-1-óxido (1) , 1-metil-3-(4-carboxifenil)triazeno-1-óxido (2), bis-1,3-(4-acetiloxima)triazeno (3), bis-1,3 (4acetilfenil)triazeno (4), 1-(2-fluorfenil)-3-(4-acetilfenil)triazeno (5), 1-fenil-3-(4-acetilfenil)triazeno (6), 1,3-bis-(4-etoxicarbonilfenil)triazeno (7), 1-(4-etoxifenil)-3-(4-carboxifenil)triazeno (8), 1-fenil-3-(4-nitrofenil)triazeno-1-óxido (9), 1-(4-nitrofenil)-3-(4-carboxifenil)triazeno (10), 1-(4acetilaminofenil)-3-(4-carboxifenil)triazeno (11), 1,3-(3-hidróxi-difeniltriazenido)(piridil)(bis-oxo-vanádio)(v) (12), Complexo 3-(4carboxilatofenil)-1-metiltriazeno 1-óxido de potássio tetraidratado (13), 1-(3-nitrofenil)-3-feniltriazeno (14), 1-(4-nitrofenil)-3-benziltriazeno (15). $\mathrm{b}_{-}=$MIC maior que $128 \mu \mathrm{g} / \mathrm{mL}$

TABELA III - Atividade antibacteriana in vitro dos compostos triazenos frente a cepas bacterianas provenientes de isolamentos clínicos do HUSM

\begin{tabular}{|c|c|c|c|c|c|c|c|c|c|c|c|c|c|c|c|}
\hline \multirow[t]{2}{*}{ Isolados clínicos } & \multicolumn{15}{|c|}{ Concentração inibitória mínima (MIC) $\mu \mathrm{g} / \mathrm{mL}$} \\
\hline & $(1)^{\mathrm{a}}$ & (2) & (3) & (4) & $(5)$ & (6) & (7) & $(8)$ & (9) & (10) & (11) & (12) & (13) & (14) & (15) \\
\hline Acinetobacter baumannii & $-\mathrm{b}$ & - & - & - & - & - & - & - & - & - & - & - & - & - & - \\
\hline Acinetobacter lwoffii & - & - & 128 & - & 128 & - & - & - & - & - & - & - & - & - & - \\
\hline Ralstonia pickettii & - & - & - & - & - & - & - & - & - & - & - & - & - & - & - \\
\hline Bordetella bronchiseptica & - & - & - & - & - & - & - & - & - & - & - & - & - & - & - \\
\hline Micrococcus sp. & 128 & - & - & 128 & - & - & - & - & - & 128 & 128 & - & - & - & - \\
\hline Enterococcus sp. & - & - & - & - & - & - & - & - & - & - & - & - & - & - & - \\
\hline Staphylococcus epidermidis & - & - & - & - & - & - & - & - & - & - & 64 & 128 & - & - & - \\
\hline Staphylococcus saprophyticus & - & - & - & 128 & - & - & - & - & 128 & 64 & - & - & - & - & - \\
\hline Streptococcus agalactiae & - & 16 & - & - & - & - & - & - & - & - & - & - & - & - & - \\
\hline Streptococcus pyogenes & - & - & - & - & - & - & - & - & - & - & - & - & - & - & - \\
\hline Bacillus cereus & - & - & - & - & - & - & - & - & - & - & - & - & - & - & - \\
\hline Corynebacterium sp. & - & - & - & - & 128 & - & - & 128 & 128 & - & - & - & - & - & - \\
\hline Rhodococcus sp. & - & - & - & 128 & - & - & - & - & 64 & 64 & 64 & - & - & - & - \\
\hline Salmonella sp. & - & - & - & - & - & - & - & - & - & - & - & - & - & - & - \\
\hline Serratia marcescens & - & - & - & - & - & - & - & - & - & - & - & - & - & - & - \\
\hline Morganella morganii & - & - & - & - & - & - & - & - & - & - & - & - & - & - & - \\
\hline Enterobacter cloacae & - & - & - & - & - & - & - & - & 32 & 32 & 32 & - & - & - & - \\
\hline Shigella flexneri & - & - & - & - & - & - & - & - & - & 128 & - & - & - & - & - \\
\hline Shigella sonnei & - & - & - & - & - & - & - & - & - & 128 & - & - & - & - & - \\
\hline Shigella spp & - & - & - & - & - & - & - & - & - & - & - & - & - & - & - \\
\hline Klebsiella pneumoniae & - & - & - & - & - & - & - & - & - & - & - & - & - & - & - \\
\hline ESBL Klebsiella oxytoca 14 & - & - & - & - & - & - & - & - & - & - & - & - & - & - & - \\
\hline ESBL Klebsiella pneumoniae 23 & - & - & - & - & - & - & - & - & - & - & - & - & - & - & - \\
\hline ESBL Klebsiella pneumoniae 24 & - & - & - & - & - & - & - & - & - & - & - & - & - & - & - \\
\hline ESBL Klebsiella pneumoniae 25 & - & - & - & - & - & - & - & - & - & - & - & - & - & - & - \\
\hline ESBL Escherichia coli 26 & - & - & - & - & - & - & - & - & - & - & - & - & - & - & - \\
\hline ESBL Klebsiella pneumoniae 27 & - & - & - & - & - & - & - & - & - & - & - & - & - & - & - \\
\hline ESBL Klebsiella pneumoniae 31 & - & - & - & - & - & - & - & - & - & - & - & - & - & - & - \\
\hline ESBL Escherichia coli 32 & - & - & - & - & - & - & - & - & - & - & - & - & - & - & - \\
\hline ESBL Klebsiella pneumoniae 37 & - & - & - & - & - & - & - & - & - & - & - & - & - & - & - \\
\hline ESBL Escherichia coli 38 & - & - & - & - & - & - & - & - & - & - & - & - & - & - & - \\
\hline
\end{tabular}

a 1-metil-3-feniltriazeno-1-óxido (1), 1-metil-3-(4-carboxifenil)triazeno-1-óxido (2), bis-1,3-(4-acetiloxima)triazeno (3), bis-1,3-(4acetilfenil)triazeno (4), 1-(2-fuorfenil)-3-(4-acetilfenil)triazeno (5), 1-fenil-3-(4-acetilfenil)triazeno (6), 1,3-bis-(4-etoxicarbonilfenil) triazeno (7), 1-(4-etoxifenil)-3-(4-carboxifenil)triazeno (8), 1-fenil-3-(4-nitrofenil)triazeno-1-óxido (9), 1-(4-nitrofenil)-3-(4-carboxifenil)triazeno (10), 1-(4-acetilaminofenil)-3-(4-carboxifenil)triazeno (11), 1,3-(3-hidroxi-difeniltriazenido)(piridil)(bis-oxo-vanádio)(v) (12), 3-(4-carboxilatofenil)1-metiltriazeno 1-óxido de potássio tetraidratado (13), 1-(3-nitrofenil)-3-feniltriazeno (14), 1-(4-nitrofenil)-3-benziltriazeno (15).

$\mathrm{b}_{-}=$MIC maior que $128 \mu \mathrm{g} / \mathrm{mL}$ 
1-metil-3-( $p$-carboxifenil)triazeno 1-óxido (2) ) e o $p$ acetil-o-fluor (5) apresentaram atividade bactericida, frente a S. agalactiae $(\mathrm{CBM}=32 \mu \mathrm{g} / \mathrm{mL})$ e Corynebacterium $\mathrm{sp}$. $(\mathrm{CBM}=128 \mu \mathrm{g} / \mathrm{mL})$ respectivamente. Sugere-se como explicação do modo de ação desses compostos a sua capacidade quelante. Eles formariam quelatos com constituintes da parede celular, impedindo a síntese desta estrutura bacteriana. Essa mesma suposição foi apresentada por Goswwani e Purohit (2001) para explicar a atividade antimicrobiana de hidroxitriazenos.

\section{CONCLUSÃO}

Dos quinze compostos triazenidos testados, 12 apresentaram algum tipo de atividade antibacteriana, fato que evidencia o potencial terapêutico dos compostos triazenidos principalmente em relação às bactérias. A elucidação do modo e do mecanismo de ação desses compostos depende de estudos adicionais. Entretanto, os resultados obtidos, nesse trabalho, apontam que os TZC constituem uma promissora classe de compostos para estudos adicionais em organismos eucarióticos e animais, para avaliar sua eficácia e toxicidade, in vitro e in vivo.

\section{ABSTRACT \\ Triazenes and antibacterial activity}

Fifteen triazenes compounds were studied concerning their antibacterial activity by broth microdilution method. Minimum inhibitory concentration (MIC) and minimum bactericidal concentration (MBC) was determined with $\mathrm{E}$. coli ATCC 25922, Staphylococcus aureus ATCC 25923, Pseudomonas aeruginosa ATCC 27853, Acinetobacter baumannii, Acinetobacter lwoffii, Ralstonia pickettii, Bordetella bronchiseptica, Micrococcus sp., Enterococcus sp., Staphylococcus epidermidis, Staphylococcus saprophyticus, Streptococcus agalactiae, Bacillus cereus, Corynebacterium sp., Rhodococcus sp., Salmonella sp., Serratia marcescens, Morganella morganii, Enterobacter cloacae, Shigella flexneri, Shigella sonnei, Shigella sp., Klebsiella pneumoniae, ESBL Klebsiella oxytoca 14, ESBL Klebsiella pneumoniae 23, ESBL Klebsiella pneumoniae 24, ESBL Klebsiella pneumoniae 25, ESBL Escherichia coli 26, $E S B L$ Klebsiella pneumoniae 27, ESBL Klebsiella pneumoniae 31, ESBL Escherichia coli 32, ESBL Klebsiella pneumoniae 37 e ESBL Escherichia coli 38. The highest effect was evidenced by the compound 1-methyl-3-(pcarboxyphenyl)triazene 1-oxide (2) against Streptococcus agalactiae $(M I C=16 \mu \mathrm{g} / \mathrm{mL}$ and $M B C=32 \mu \mathrm{g} / \mathrm{mL})$. The compounds 1-phenyl-3-(4-nitrophenyl)triazene-1-oxide (9), 1-(4-nitrophenyl)-3-(4-carboxyphenyl)triazene (10) e 1-(4acethyl amine phenyl)-3-(4-carboxyphenyl)triazene (11) presented MIC between 32 and $64 \mu \mathrm{g} / \mathrm{mL}$ against $\mathrm{S}$. edipermidis, S. saprophyticus, Rhodococcus sp. and E. cloacae. The compounds 1-methyl-3-phenyltriazene-1-oxide (1) , bis-1,3-(4-acethyl oxime)triazene (3), bis-1,3 (4acethyl phenyl)triazene (4), 1-(2-fluorophenyl)-3-(4acethylphenyl)triazene (5), 1,3-(3-hydroxy-diphenyltriazenide)(piridil)(bis-oxo-vanadium) (12), 1-(3nitrophenyl)-3-phenyltriazene (14), 1-(4-nitrophenyl)-3benzyltriazene (15) presented MIC of the $128 \mu \mathrm{g} / \mathrm{mL}$ against $\mathrm{S}$. aureus ATCC 25923, P. aeruginosa ATCC 27853, A. lwoffii, Micrococcus sp., S. epidermidis, S. saprophyticus, Corynebacterirum sp., E. cloacae, S. flenneri e S. sonnei. The compounds 1-phenyl-3-(4-acethylphenyl)triazene (6), 1,3-bis-(4-ethoxycarbonylphenyl) triazene (7), and 3-(4-carboxylatephenyl)-1-methyltriazene 1-oxide of potassium tetrahydrate (13) presented MICs equals or highest than $128 \mu \mathrm{g} / \mathrm{mL}$. The results have demonstrated the potential biological activity of these compounds against Gram-positive and Gram-negative bacteria.

UNITERMS: Triazenes/antibacterial activity. Broth microdilution method.

\section{AGRADECIMENTOS}

M.H. agradece ao CNPq (Bolsa de Produtividade em pesquisa). R.H. agradece bolsa FIPE Júnior/UFSM.

\section{REFERÊNCIAS BIBLIOGRÁFICAS}

BRASIL. Ministério da Saúde. Agência Nacional de Vigilância Sanitária. Manual de microbiologia clínica para o controle de infecção em serviços de saúde: Brasília: ANVISA, 2004. Módulos I, p.65; II, p.37; III, p.43; IV, p.64 e V, p.93.

CARSON, C., NARBER, K. G. Role of fluorquinolones in treatment of serious bacterial urinary tract infections. Drugs, v.64, p.1359-1373, 2004.

CARVALHO, E.; FRANCISCO, A. P.; ILEY, J.; ROSA, E. Triazene drug metabolites. Part 17: Synthesis and plasma hydrolysis of acyloxymethyl carbamate derivatives of antitumour triazenes. Bioorg. Med. Chem., v.8, p.1719$1725,2000$. 
CLINICAL AND LABORATORY STANDARDS INSTITUTE. Methods for dilution antimicrobial susceptibility tests for bacteria that grow aerobically. Approved Standard. 6.ed. Wayne: NCCLS, 2003. p.53. (NCCLS document M7-A6).

COUDRON, P. E.; HANSON, N. D.; CLIMO, M. W. Occurrence of extended-spectrum and AmpC betalactamases in bloodstream isolates of Klebsiella pneumoniae: isolates harbor plasmid-ediated FOX-5 and ACT-1 AmpC Beta-Lactamases. J. Clin. Microbiol., v.41, p.772-777, 2003.

GIGLIO, V. F. Síntese e cristaloquímica de complexos de $\mathrm{Hg}$ (II) e Ni(II) com o ligante 3-(2-fluorofenil)-1-(4acetilfenil)triazenido e atividade biológica de triazenos livres. Santa Maria, 2006. 135 f. [Dissertação de Mestrado. Universidade Federal de Santa Maria].

GOSWAMI, A. K.; PUROHIT, D. N. Synthesis and antimicrobial activities of some hydroxytriazenes: a new class o biologically active compounds. Anal. Sci., v.17, p.1789-1791, 2001.

HÖRNER, M.; BRANCHER, A.L.; SILVA, A.; BORDINHÃO, J.; MOESSMER, C. M. 3-(4Acetylphenyl)-1-(4-nitrophenyl)-triazene. Acta Crystallogr., Sect. C: Cryst. Struct. Commun, v.C60, p.303-304, 2004.

HÖRNER, M.; BRESOLIN, L.; BORDINHÃO, J.; HARTMANN, E.; STRÄHLE, J. 1,3-Bis(4nitrophenyl)triazene. Acta Crystallogr., Sect. C: Cryst. Struct. Commun, v.59, p.426-427, 2003.

HÖRNER, M.; OLIVEIRA, G. M.; BEHM, M. B.; FENNER, H. Bidimensional self-assembling of $\left[\mathrm{Hg}^{\mathrm{II}}[\mathrm{RPhNNNPhR0})_{2}\right]\left(\mathrm{R}=\right.$ acetyl, $\left.\mathrm{R}^{\prime}=\mathrm{F}\right)$ through metal- $\eta^{4}$-arene $\pi$-interactions and non classical $\mathrm{C}-\mathrm{H} \cdots \mathrm{O}$ bonding: Synthesis and X-Ray characterization of a bis diaryl symmetric-substituted triazenide complex polymer of $\mathrm{Hg}(\mathrm{II})$. Z. Anorg. Allg. Chem., v.632, p.615-618, $2006 \mathrm{a}$

HÖRNER, M.; OLIVEIRA, G. M.; GIGLIO, V. F.; VISENTIN, L. C.; BROCH, F.; BECK, J. Inorg. Chim. Acta, v.359, p.2309, 2006b.

HÖRNER, R. Estudo de compostos capazes de clivar o DNA. Florianópolis, 2003. Número de páginas? [Tese de Doutorado. Universidade Federal de Santa Catarina]. p.238.
HÖRNER, M.; OLIVEIRA, J. S.; BORDINHÃO, J.; BECK, J. trans-Bis[1-methyl-3-( $p$-nitrophenyl)triazenido 1oxide-: $\left.{ }^{2} N^{3}, O\right]$ dipyridinenickel(II). Acta Crystallogr., Sect. $C$, v.C58, p.m586-m587, 2002.

JONES, R. N.; PFALLER, M. A. Antimicrobial activity against strains of Escherichia coli and Klebsiella sp. with resistance phenotypes consistent with an extendedspectrum $\beta$-lactamase in Europe. Clin. Microbiol. Infect., v.9, p.708-712, 2003.

JACKSON, L. A.; HILSDON, R.; FARLEY, M. M.; HARRISON, L. H.; REINGOLD, A. L.; PLIKAYTIS, B. D.; WENGER, J.D.; SCHUCHAT, A. Risk factors for group B streptococcal disease in adults. Ann. Intern. Med., v.123, p.415-420, 1993.

LIVERMORE, D. M. Mechanisms of resistance to ${ }^{2}$-lactam antibiotics. Scand. J. Infect. Dis., v.78, p.7-16, 1991.

MURRAY, P. R.; BARON, E. J.; PFALLER, M. A.; TENOVER, F. C.; YOLKEN, R. H. M. Manual of clinical microbiology. 8. ed. Washington: ASM Press, 2003. p. 2113.

MURRAY, R. P.; ROSENTHAL, K. S.; KOBAYASHI, G. S.; PFALLER, M. A. Microbiologia médica. 4 ed. Rio de Janeiro: Guanabara Koogan, 2004. p. 776.

PAGANI, L.: DELL'AMICO, E.; MIGLIAVACCA, R.; D'ANDREA, M. M.; GIACOBONE, E.; AMICOSANTE, G.; ROMERO, E.; ROSOLINI, G. M. Multiple CTX- $M$-type extended-spectrum $ß$-lactamases in nosocomial isolates of Enterobacteriaceae from a hospital in northern Italy. J. Clin. Microbiol., v.41, p.4264-4269, 2003.

PARAGINSKI, G. L.; REETZ, L. G. B.; MICHELOT, T. M.; DOMINGUES, V. O.; BERTONCHELI, C. M.; MARASCHIN, M. M.; VISENTIN, L. C.; SILVA, A.; GIGLIO, V. F. WETSPHALEN, A. B.; AMARAL, C. H. O.; HÖRNER, M.; HÖRNER, R. Avaliação da atividade de clivagem do DNA plasmidial por cinco compostos triazenos inéditos. In: CONGRESSO BRASILEIRO DE ANÁLISES CLÍNICAS, 33.; CONGRESSO BRASILEIRO DE CITOLOGIA CLÍNICA, 6., 2006, Curitiba. Resumos. Rio de Janeiro: Sociedade Brasileira de Análises Clinicas, 2006. p.90B, res. n.363. 
PARODI, S.; LECHNER, A.; OSIH, R.; VESPA, P.; PEGUES, D. Nosocomial Enterobacter meningitis: risk factors, management, and treatment outcomes. Clin. Infect. Dis., v.15, p.159-166, 2003.

PEORI, M. B.; VAUGHAN, K.; HOOPER, D.L. Synthesis and Characterization of Novel Bis-Triazenes: 3,8-Di[2aryl-1-azenyl]-1,3,6,8-tetraazabicyclo[4.4.1]undecanes and 1,3-Di-2-[(4-methoxyphenyl)-1diazenyl]imidazolidine. The reaction of diazonium ions with ethylenediamine/formaldehyde mixtures. $J$. Org. Chem., v.63, p.7437-7444, 1998.

PEREIRA, A. S.; CARMO FILHO, J. R.; TOGNIM, M. C. B.; SADER, H. S. Avaliação da acurácia de testes laboratoriais para detecção de amostras de Klebsiella pneumoniae produtora de ${ }^{2}$-lactamase de espectro ampliado. J. Bras. Patol. Med. Labor., v.39, p.301-308, 2005.

PILCH, D. S.; KIROLOS, M. A.; LIU, X.; PLUM, G. E.; BRESLAUER, K. J. Berenil [1,3-bis-(4amidinophenyl)triazene] binding to DNA duplexes and a RNA duplex: evidence for both intercalative and minor groove binding properties. Biochemistry, v.34, p.99629976, 1995.

SADER, H. S.; GALES, A. C.; PFALLER, M. A.; MENDES, R. E.; ZOCCOLI, C.; BARTH, A.; JONES, R. N. Pathogen frequency and resistance patterns in Brazilian hospitals: summary of results from three years of the SENTRY antimicrobial surveillance program. Braz. J. Clin. Microbiol., v.5, p.200-214, 2001.

SANADA, M.; TAKAGI, Y.; SEKIGUCHI, M. Killing and mutagenic actions of dacarbazine, a chemotherapeutic alkylating agent, on human and mouse cells: effects of Mgmt and Mlh1 mutations. DNA repair, v.3, p.413-420, 2004.
SANDERS, W. E.; SANDERS, C. C. Enterobacter sp.: pathogens poised to flourish at the turn of the century. Clin. Microbiol. Rev., v.10, p.220-241, 1997.

SANTOS, A. J. R. W. A. Síntese, estruturas de triazeno 1-óxido e complexo de $\mathrm{K}^{+}$, e avaliação da atividade biológica de 1-metiltriazeno 1-óxido e 1,3bis(aril)triazenos. Santa Maria, 2005. p.53 [Dissertação de Mestrado. Universidade Federal de Santa Maria].

SCHUCHAT, A. Epidemiology of group B streptococcal disease in the United States: shifting paradigms. Clin. Microbiol. Rev., v.11, p.497-513, 1998.

SHEALY, Y. F.; KRAUTH; C. A.; PITTILLD, R. F.; HUNT, D. E. A new antifungal and antibacterial agent, methyl 5(or 4)-(3,3-dimethyl-1-triazeno)imidazole-4(or 5)carboxylate. J. Pharm. Sci., v.15, p.147-148, 1966.

TAKAHASHI, H.; KRAMER, M. H.; YASUI, Y.; FUJII, H.; NAKASE, K.; IKEDA, K.; IMAI, T.; OKAZAWA, A.; TANAKA, T.; OHYAMA, T.; OKABE, N. Nosocomial Serratia marcescens outbreak in Osaka, Japan, from 1999 to 2000. Infect. Control. Hosp. Epidemiol., v.25, p.156$161,2004$.

TRAUTNER, B. W.; DAUROWCH, R. O. Role of biofilm in catheter-associated urinary tract infection. Amer. $J$. Infect. Control, v.32, p.177-183, 2004.

ZAR, H. J.; COTTON, M. F. Nosocomial pneumonia in pediatric patients: practical problems and rational solutions. Ped. Drugs, v.4, p.73-83, 2002.

ZHAO, S.; XIA, X,; XIANGWEN, K.; LIU, T. Highly sensitive colour-reaction of nickel with a new chromogenic reagent benzothiaxolyldiazoaminoazobenzene and its application. Talanta, v.45, p.13-17, 1997.

Recebido para publicação em 13 de junho de 2007 Aceito para publicação em 19 de abril de 2008 